\title{
Preprocessing and Enhancement for Image Fusion using Composite Algorithm
}

\author{
Shirish Kulkarni ${ }^{1^{*}}$, and Bhavesh Digey ${ }^{1^{*}}$ \\ ${ }^{1}$ Department of Instrumentation Engineering, Ramrao Adik Institute of Technology, Navi Mumbai
}

\begin{abstract}
A technique of alignment and removal of noise is a prominent pre-processing part of biomedical image fusion. The purpose is to evaluate and analyze visually as well as parametric findings. However, considering practical execution still there is need for improvement in pre-processing for image processing applications. Therefore, the problem of blockage and variation in scans of patients, need to be overcome by properly align and denoise input images. Therefore to design the registration algorithm in such a way, it should cover all geometric motions of biomedical images, by which it is useful to the practitioner for the detection of medical defects. Herewith we have proposed a log-polar and phase correlation composite algorithm for the registration of all geometric motions. The proposed algorithm preserves the outline portion and surface information from the images, which yields perceptible effects. This will be useful in order to take out visual data from a noisy background. Since it is shown that the results with noisy and after denoised compared visually and also parametric analysis is carried out by calculating PSNR, MSE, contrast, structural content, entropy, etc
\end{abstract}

\section{INTRODUCTION}

Image registration is a process of mapping a sensed image with the reference image that shares common visual information such as medical images belonging to the similar object or images of the same scene obtained from different viewpoints, or at different time instances, or sensors. However, the objective of such mapping is to match image registration, which is quintessential in many image processing applications, that incorporates a number of images for the purpose of comparison, integration. The main objective of the image registration process is to estimate the geometric transformations of the reference image IR to the sensed image, IS, where $\operatorname{IS}(x, y)=T\left\{\operatorname{IR}\left(x^{\prime}, y^{\prime}\right)\right.$ and $T$ is a 2-D geometric transformation that includes scale, rotation, and translation in the Cartesian coordinates. Image registration technique should comprise of geometric transformation of all motions along. There should be accountability with denoising removal of noise present in sense image due to motion artifacts before applying the transformation. The proposed method not only preserves edges and texture information from the image but also removes the noise, providing the good visual effect.

It is very important to have a low computation cost with respect to the time and load of the matrix formation. The size and correctness of the images rely on the number of samples taken into consideration. For the registration of any medical image, the scaled mapping in terms of radius and angular direction is required. This is the main cause of increases the load on computation. To increase the effectiveness and robustness of the changes, we proposed the area based Log-Polar Transformer (LPT) method. This method again increases the computational load during the mapping process. This will motivate the success of area-based LPT -PC composite algorithm this will scale up the all the geometric motion. The image registration using composite algorithm is used to get results with low computation cost.

The rest of paper is organized as follows; in the first section details of geometric actions and their methodology using Log-Polar transform and Phase correlation is explained. Literature survey is included which accomplishes work on denoising and registration. Implementation of image registration is carried out by the proposed algorithm in the consecutive section. In the next section, performance evaluation is carried on medical images (CT scan) with visual as well as parametric analysis. The last section concludes the work.

\section{LITERATURE SURVEY}

The various image registration approaches are proposed in the literature. The SIFT, SURF, and LPT are the major approaches. Scale Invariant Feature Transform (SIFT) enables the extraction of invariant features in a pyramidal Difference of Gaussians (DOG) and may render itself invariant to image scaling, translation, and rotation, and to some extent also invariant to

\footnotetext{
* Corresponding author: shirish.kulkarni@rait.ac.in, bhavesh.digey@,rait.ac.in
} 
illumination changes and affine [1-3]. Principal Components Analysis (PCA) is used to standardize the gradient patch, as a substitute of SIFTs sharp weighted histograms. The SIFT improves the feature matching and splits the extracted features into some sub-collections before the two images are matched [4-5].

SURF (Speeded-up Robust Features) constructs pyramids of different resolutions, uses a different method to extract feature points. It relies on the integral images for image convolutions. SURF is faster than SIFT but both aren't robust when it comes to rotation and illumination changes [6].

The similarities between the images in the frequency domain are evaluated by using the phase correlation method [7]. As compared to cross-correlation, it performs well with noise and illumination changes. The correlation method succumbs to failure when there are changes in rotation or scale between images, in such cases, Log-Polar Transform (LPT) [8-13] is widely used. Fourier-Mellin used a composite algorithm (phase correlation technique with the Log-Polar Transformation), it describes rotation and scale as translations in parameter space [14-17].

\section{PROPOSED IMAGE REGISTRATION METHOD}

For spinning and zooming geometric effects in image Log-Polar Transform technique is used. In this method, angular and scaling effect occurs as translation. The polar coordinates $(\rho, \theta)$ aligns with the radius and angle from the center. This approach can be evaluated by taking into consideration, logarithm of points placed in the circle $(\rho)$. The scale transform is insensible to enlarge and pertain to scale energy spectrum distingusihes both regardless of the magnification factor. Denoising caried out in the scale domain is promising than in Fourier domain which was indicated by Moses as its importance in extracting significant information from a background of distorted images. The advantage of scale transform is that it enables us to smooth-out the spectrum without spreading the scales. It is seen that in log-polar images parameters associated with translation motion are not successfully obtained, this can be overcomed by phase correlation method.

The block diagram shown in fig. 1 is of proposed method consisting of input images to be registered, transformation for geometric motions followed by sense image aligned on the reference image to obtain a registered image. The major difference in block diagram from rest is the occurrence of image denoising using the same transform that will be used for geometric transformations.

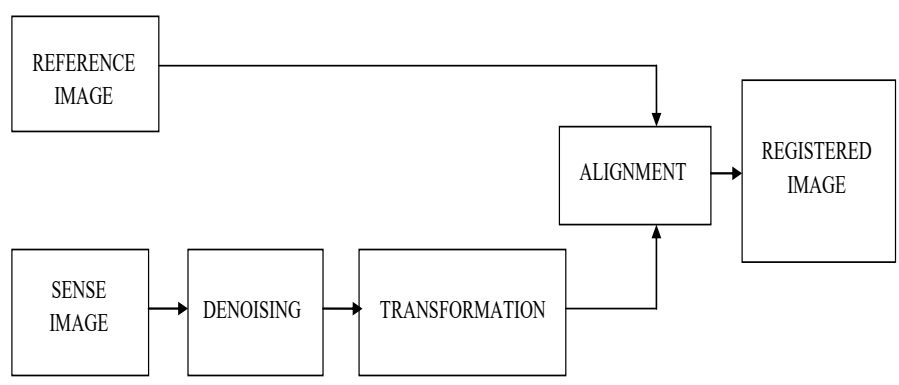

Fig 1. Block Diagram of Proposed Image Registration Technique

The proposed registration algorithm is as follow: We have two source images $I i, i=1,2$ related by a translation, rotation, and scaling.

1. Calculate the gradient as $\mathrm{Gi}=\mathrm{Gi}(\mathrm{x})+\mathrm{j} \mathrm{Gi}(\mathrm{y})$

2. Horizontal and Vertical boundaries are obtained using the regular Cartesian FFT by using a high pass emphasis filter.

3. Removal of noise components from the sensed image using log-polar transform.

4. Estimate rotation $(\theta)$ and scale (s) using the logpolar technique.

5. Scale up/down and de-rotate the image with respect to estimated angle.

6. Evaluate the transformation with the Phase Correlation in a frequency domain.

7. Then IFFT is taken and calculate the peaks for both cases.

8. Now for images such as reference image $\mathrm{I}^{\mathrm{R}}$ and sense image $\mathrm{I}^{\mathrm{s}}$, compute Cross Power Spectrum with the reference image.

9. As in step 5, shift the image to the original position and finally, this gives a registered image.

\section{RESULTS AND DISCUSSION}

Performance analysis has been carried out for all four cases and the results at each case are evaluated and explained below. Image registration for chest, abdomen, lungs and head CT of four different cases are carried out and visual analysis of all four cases are shown with noise and after denoising effect shown in fig. $2,4,6,8$ and 3,5,7,9 respectively. Performances of all the cases are improved with denoising effect. Hence there is a need for pre-processing before transforming sense image. Also with the parametric analysis from table 1, 2, 3 and 4, it is observed that there is an improvement in Mean Squared Error (MSE) and Peak Signal to Noise Ratio (PSNR) with denoising effect i.e there is an increase in PSNR and decrease in MSE respectively. 

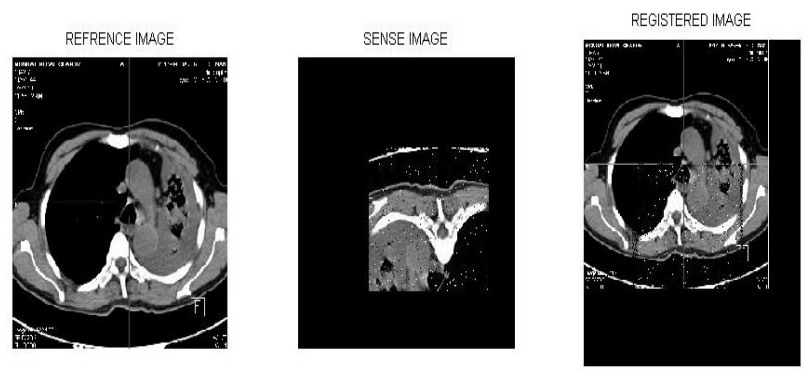

Fig 2. Image Registration for Chest CT with Noise
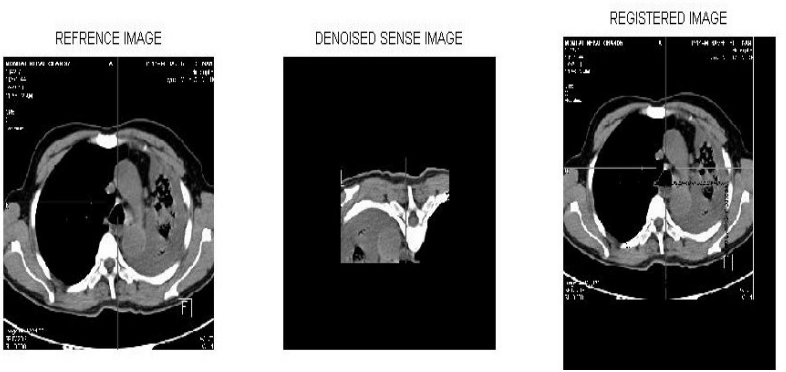

Fig 3. Image Registration for Chest CT with Denoise effect Horizontal movement: 79, Vertical movement: 43, Rotated angle: $180^{\circ}$

The composite algorithm applied on Head CT has successfully registered for sense image with partial data, without exact angle shown in figure 10 and scaling effect shown in figure 11.

Table 1. Chest CT: Comparision of registered image with and without denoising effect

\begin{tabular}{|c|c|c|c|}
\hline $\begin{array}{c}\text { Sr. } \\
\text { No. }\end{array}$ & Parameters & $\begin{array}{c}\text { With } \\
\text { Noise }\end{array}$ & $\begin{array}{c}\text { With } \\
\text { Denoising }\end{array}$ \\
\hline 1 & Standard Deviation & 96.32 & 96.92 \\
\hline 2 & Structural Content & 2.316 & 2.406 \\
\hline 3 & MSE & 26.26 & 20.59 \\
\hline 4 & PSNR & 33.97 & 35.02 \\
\hline 5 & Contrast & 1.0744 & 1.117 \\
\hline 6 & Entropy & 4.807 & 4.61 \\
\hline
\end{tabular}
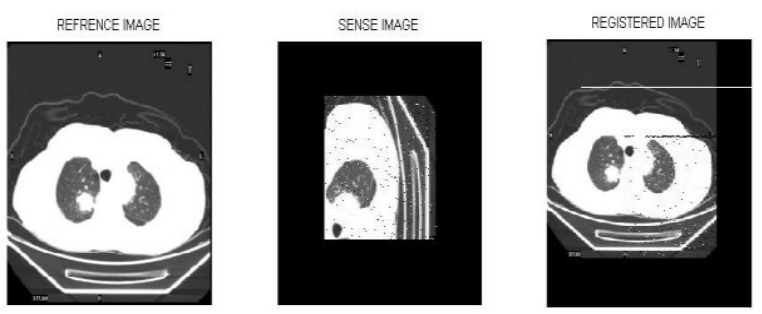

Fig 4. Image Registration for Abdomen CT with Noise effect
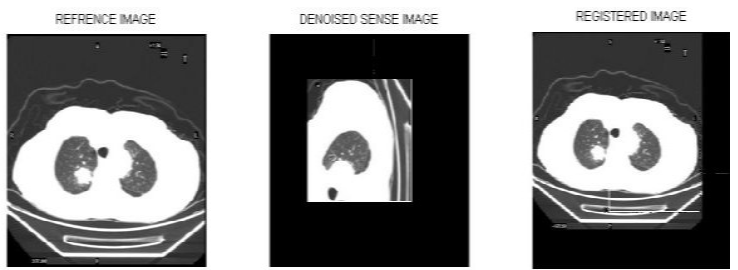

Fig 5. Image Registration for Abdomen CT with Denoise effect

Horizontal movement: 55, Vertical movement: 51, Rotated angle: $90^{\circ}$
Table 2. Abdomen CT: Comparision of registered image with and without denoising effect

\begin{tabular}{|c|c|c|c|}
\hline $\begin{array}{c}\text { Sr. } \\
\text { No. }\end{array}$ & Parameters & $\begin{array}{c}\text { With } \\
\text { Noise }\end{array}$ & $\begin{array}{c}\text { With } \\
\text { Denoising }\end{array}$ \\
\hline 1 & Standard Deviation & 66.27 & 66.295 \\
\hline 2 & Structural Content & 2.436 & 2.959 \\
\hline 3 & MSE & 26.261 & 20.592 \\
\hline 4 & PSNR & 33.971 & 35.027 \\
\hline 5 & Contrast & 1.82 & 1.908 \\
\hline 6 & Entropy & 3.819 & 3.77 \\
\hline
\end{tabular}
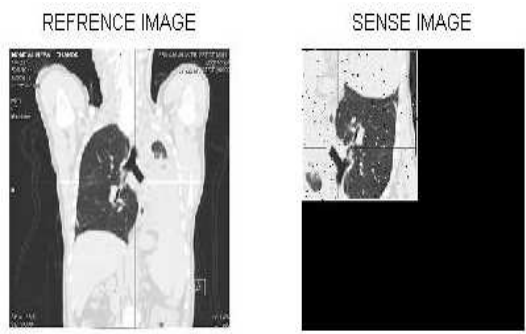

REGISTERED IMAGE

Fig 6. Image Registration for Lungs CT with Noise effect REFRENCE IMAGE

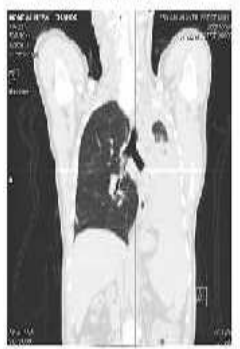
SENSE IMAGE

REGISTERED IMAGE
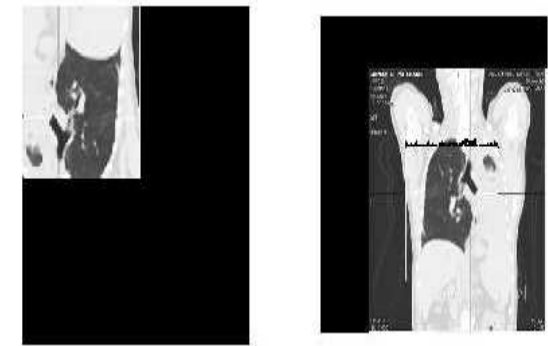

Fig 7. Image Registration for Lungs CT with Denoise effect Horizontal movement: - 44, Vertical movement: -70, Rotated angle: $180^{\circ}$.

Table 3. Lungs CT: Comparision of registered image with and without denoising effect

\begin{tabular}{|c|c|c|c|}
\hline $\begin{array}{c}\text { Sr. } \\
\text { No. }\end{array}$ & Parameters & $\begin{array}{c}\text { With } \\
\text { Noise }\end{array}$ & $\begin{array}{c}\text { With } \\
\text { Denoising }\end{array}$ \\
\hline 1 & Standard Deviation & 101.46 & 101.72 \\
\hline 2 & Structural Content & 3.10 & 3.223 \\
\hline 3 & MSE & 57.31 & 55.72 \\
\hline 4 & PSNR & 30.58 & 30.704 \\
\hline 5 & Contrast & 0.99 & 1.008 \\
\hline 6 & Entropy & 5.37 & 5.189 \\
\hline
\end{tabular}

REFAEICE MAGGE

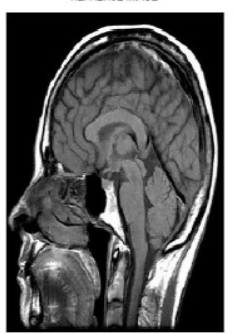

Fig 8. Image Registration for Head CT with Noise effect
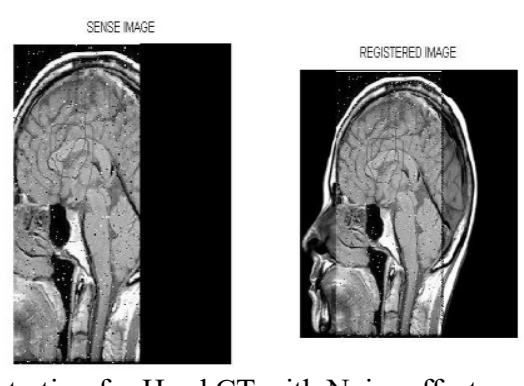

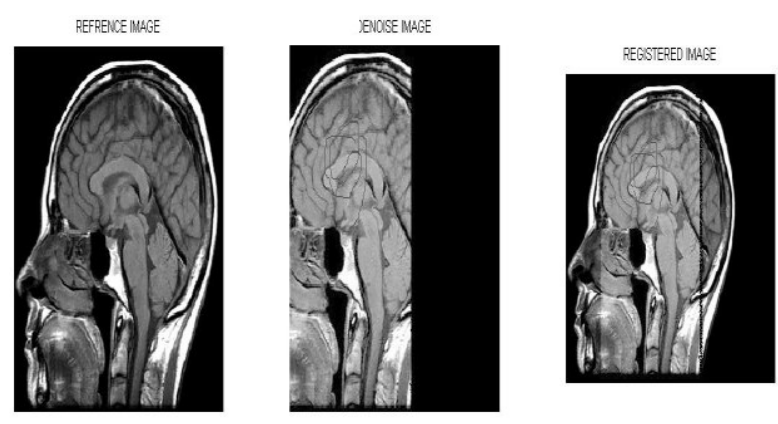

Fig 9. Image Registration for Head CT with Denoise effect Horizontal movement: 01,Vertical movement: 50, Rotated angle: $0^{\circ}$

Table 4. Head CT: Comparision of registered image with and without denoising effect

\begin{tabular}{|c|c|c|c|}
\hline $\begin{array}{c}\text { Sr. } \\
\text { No. }\end{array}$ & Parameters & With Noise & $\begin{array}{c}\text { With } \\
\text { Denoising }\end{array}$ \\
\hline 1 & Standard Deviation & 78.987 & 80.867 \\
\hline 2 & Structural Content & 0.94 & 0.96 \\
\hline 3 & MSE & 81.386 & 79.458 \\
\hline 4 & PSNR & 29.059 & 29.163 \\
\hline 5 & Contrast & 1.025 & 1.071 \\
\hline 6 & Entropy & 5.907 & 5.630 \\
\hline & & & \\
\hline
\end{tabular}

Fig 10. Image Registration for Head $C T$ with rotation without an exact angle.

Horizontal movement: - 42, Vertical movement: 7, Rotated angle: $203^{\circ}$
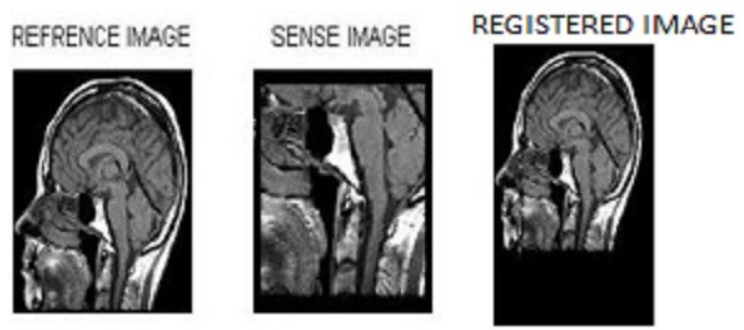

Fig 11. Image Registration for Head CT with scale. Estimated Horizontal movement 103, Vertical movement 44, Scale 1.57

\section{CONCLUSION}

The manuscript presents a comparison between noisy and denoised registered images of different CT images of human body organs like abdomen, chest, lungs, and head. The proposed algorithm preserves edges and texture information from the sensed CT images and also removes the noise, providing the good visual effect.
This will be useful in order to take out visual data from a noisy background.

The simulated results of image registration are verified with scaling factor up to 1.57 and with any degree of rotation. The accuracy of the algorithm is verified by visual inspection of the registered image by overlaying it on a reference image. Moreover, the parametric analysis is carried out between the registered and reference images by calculating PSNR, MSE, contrast, structural content, entropy, etc.. The facility of the algorithm to improve registration parameters in presence of noise, partial data and with blurring is also confirmed. The simulated results show that this approach is highly effective.

\section{References}

1. David G. Lowe. Object recognition from local scaleinvariant features, In Proceedings of the Seventh IEEE International Conference on Computer Vision, Corfu, Greece, 1999.

2. Yu-Dae Ban, Jaeyeon Lee, Dae Hwan Hwang, YunKoo Chung. Face Image registration methods using Normalized Cross Correlation International Conference on Control. Automation and Systems, 2008, pp.2408-2411.

3. David G. Lowe. Distinctive image features from scaleinvariant key- points. International Journal of Computer Vision, 2004, (60), pp. 91-110.

4. Yan Ke and R. Sukthankar. PCA-SIFT: a more distinctive representation for local image descriptors. In Proceedings IEEE Computer Society Conference on Computer Vision and Pattern Recognition. 2004 July, 2(2), pp. 506-513.

5. Danijela Ristic-Durrant Faraj Alhwarin, Chao Wang, and Axel Grser. Improved sift features matching for object recognition. BCS International Academic Conference 2008 Visions of Computer Science. 2008, pp. 179-190.

6. Luo Juan and Oubong Gwon. A Comparison of SIFT, PCA-SIFT, and SURF. International Journal of Image Processing (IJIP). 2009, 3(4), pp. 143-152.

7. Jignesh N Sarvaiya, Dr. Suprava Patnaik, Salman Bombaywala. Image registration using Log polar transform and phase correlation. IEEE Region 10 Conference TENCON. 2009, pp. 1-5.

8. Hast, Anders. Robust and Invariant Phase Based Local Feature Matching. 22nd International Conference on Pattern Recognition (ICPR). 2014, ISSN: 1051-4651, pp. 809-814.

9. Wolberg G., Zokai S, Robust image registration using log-polar transform, In Proceeding International Conference on Image Processing, 2000 September, pp. 493-496.

10. Y.Keller, A.Averbuch, M.Israeli. Pseudo polarbased estimation of large translations, rotations, and scalings in images. IEEE Trans. on Image processing. 2005, pp. 12-22.

11. H. Liu, B. Guo, and Z. Feng. Pseudo-log-polar Fourier transforms for image registration. IEEE 
Transaction Signal Processing Letters. 2006 January, 13(1), pp. 1720.

12. R. Matungka, Y. F. Zheng, and R. L. Ewing. 2D invariant object recognition using log-polar transform. In Proc. World Congress on Intelligent Control and Automation, 2008 January, pp. 223-228.

13. A. N. Myrna, M. G. Venkateshmurthy, and C. G. Patil. Detection of region duplication forgery in digital images using wavelets and log-polar mapping. In Proc. Intl. Conf. Computational Intelligence and Multimedia Applications, 2007 December, (3), pp. 371-377.

14. Xiaoxin Guo, Zhiwen $\mathrm{Xu}$, Yinan $\mathrm{Lu}$, and Yunjie Pang. An Application of Fourier-Mellin Transform in Image Registration. Proceedings of The Fifth International Conference on Computer and Information Technology, 2005, pp.619-623.

15. B. Srinivasa Reddy and B. N. Chatterji. An FFTBased Technique for Translation, Rotation, and ScaleInvariant Image Registration. IEEE Trans. on Image Processing. 1996, (5), pp.1266-1271.

16. XiaoxinGuo, ZhiwenXu, Yinan $\mathrm{Lu}$, and Yunjie Pang. An Application of Fourier-Mellin Transform in Image Registration. Proceedings of The Fifth International Conference on Computer and InformationTechnology, 2005, pp.619-623.

17. Y.Keller, A.Averbuch, M.Israeli. Pseudo polarbased estimation of large translations, rotations, and scalings in the image. IEEE transaction on Image processing. 2005, pp. 12-22. 